\title{
A Note on Partial Rectangular Metric Spaces
}

\author{
NGuyen Van Dung and Vo Thi Le Hang
}

\begin{abstract}
In this paper, we present a rectangular metric from a partial rectangular metric and state some relations between them. As applications, we show that fixed point theorems on partial rectangular metric spaces may be deduced from fixed point theorems on rectangular metric spaces.
\end{abstract}

\section{INTRODUCTION}

In [9], Matthews introduced the notion of a partial metric space as a part of the study of denotational semantics of data flow networks. The main difference comparing to the standard metric is that the self-distance of an arbitrary point need not be equal to zero. Many fixed point results on partial metric spaces were stated and generalized, see $[8,13,14]$ and references therein. In [2], Branciari introduced a generalized metric which was called a rectangular metric in [12]. Recently, Shukla generalized the concept of rectangular metric space and extended the concept of partial metric space by introducing the partial rectangular metric space in [12]. In that paper, a fixed point theorem for quasi type contraction was also proved in the partial rectangular metric space which generalizes several known results in metric, partial metric and rectangular metric spaces. Also, results were illustrated by some examples.

For the relation between metric spaces and partial metric spaces, some authors proved that several fixed point generalizations to partial metric spaces can be obtained from the corresponding results in metric spaces, see $[3,10,11]$.

On rectangular metric spaces, many fixed point theorems were stated in recent times, see $[4,5,6]$ and references therein. Some minor facts on the relation between rectangular metric spaces and metric spaces were pointed out in [1].

In this paper, we investigate the relation between partial rectangular metric spaces and rectangular metric spaces. We first present a rectangular

2010 Mathematics Subject Classification. Primary: 47H10, 54H25; Secondary: 54D99, $54 \mathrm{E} 99$.

Key words and phrases. Partial rectangular metric space, rectangular metric space, fixed point. 
metric from a partial rectangular metric and state some relations between them. Then we show that fixed point theorems on partial rectangular metric spaces may be deduced from certain fixed point theorems on rectangular metric spaces.

\section{Preliminaries}

We recall some notions and results which will be used in the latter.

Definition 1 ([2], Definition 1.1). Let $X$ be a non-empty set and $d: X \times$ $X \longrightarrow \mathbb{R}$ be a function. Then $(X, d)$ is called a g.m.s if the following conditions hold for all $x, y \in X$.

(1) $d(x, y) \geq 0$ and $d(x, y)=0$ if and only if $x=y$;

(2) $d(x, y)=d(y, x)$;

(3) $d(x, y) \leq d(x, w)+d(w, z)+d(z, y)$ for all distinct points $w, z \in$ $X \backslash\{x, y\}$.

For convenience, the pair $(X, d)$ is called a rectangular metric space and $d$ is called a rectangular metric in the sense of [12].

Definition 2 ([2], Definition 1.2). Let $(X, d)$ be a rectangular metric space.

(1) A sequence $\left\{x_{n}\right\}$ is called to converge to a point $x \in X$ if $\lim _{n \rightarrow \infty} d\left(x_{n}, x\right)=$ 0 , written $\lim _{n \rightarrow \infty} x_{n}=x$.

(2) A sequence $\left\{x_{n}\right\}$ is called Cauchy if $\lim _{n, m \rightarrow \infty} d\left(x_{n}, x_{m}\right)=0$.

(3) $(X, d)$ is called complete if each Cauchy sequence $\left\{x_{n}\right\}$ is convergent.

Definition 3 ([12], Definition 3). Let $X$ be a non-empty set and $p: X \times$ $X \longrightarrow \mathbb{R}$ be a function. Then $p$ is called a partial rectangular metric on $X$ if the following conditions hold for all $x, y \in X$.

(1) $p(x, y) \geq 0$

(2) $x=y$ if and only if $p(x, y)=p(x, x)=p(y, y)$;

(3) $p(x, x) \leq p(x, y)$;

(4) $p(x, y)=p(y, x)$;

(5) $p(x, y) \leq p(x, w)+p(w, z)+p(z, y)-p(w, w)-p(z, z)$ for all distinct points $w, z \in X \backslash\{x, y\}$.

The pair $(X, p)$ is called a partial rectangular metric space.

Definition 4 ([12], Definition 4$)$. Let $(X, p)$ be a partial rectangular metric space.

(1) A sequence $\left\{x_{n}\right\}$ is called to converge to a point $x \in X$, written $\lim _{n \rightarrow \infty} x_{n}=x$, if $\lim _{n \rightarrow \infty} p\left(x_{n}, x\right)=p(x, x)$.

(2) A sequence $\left\{x_{n}\right\}$ is called Cauchy if $\lim _{n, m \rightarrow \infty} p\left(x_{n}, x_{m}\right)$ exists and is finite. 
(3) $(X, p)$ is called complete if for each Cauchy sequence $\left\{x_{n}\right\}$, there exists $x \in X$ such that

$$
\lim _{n, m \rightarrow \infty} p\left(x_{n}, x_{m}\right)=\lim _{n \rightarrow \infty} p\left(x_{n}, x\right)=p(x, x) .
$$

Note that every rectangular metric is a partial rectangular metric. Some interesting examples of partial rectangular metrics are as follows.

Example 1 ([12], Example 1). Let $X=[0, a]$ and $b \geq a \geq 3$ and

$$
\rho(x, y)= \begin{cases}x, & \text { if } x=y \\ \frac{3 b+x+y}{2}, & \text { if } x, y \in\{1,2\}, x \neq y \\ \frac{b+x+y}{2}, & \text { otherwise. }\end{cases}
$$

Then $\rho$ is a partial rectangular metric which is not a rectangular metric.

Example 2 ([12], Proposition 3). For any rectangular metric space $(X, d)$ and constant $\alpha>0$, put $\rho(x, y)=d(x, y)+\alpha$ for all $x, y \in X$. Then $\rho$ is a partial rectangular metric which is not a rectangular metric.

Definition 5. (1) [7] Let $(X, d)$ be a rectangular metric space and $T$ : $X \longrightarrow X$ be a map. Then $T$ is called a quasi-contraction if there exists $q \in[0,1)$ such that

$$
\begin{aligned}
& d(T x, T y) \leq q \max \{d(x, y), d(x, T x), d(y, T y), d(x, T y), d(y, T x)\} \\
& \quad \text { for all } x, y \in X .
\end{aligned}
$$

(2) $[12$, Definition 5] Let $(X, p)$ be a partial rectangular metric space and $T: X \longrightarrow X$ be a map. Then $T$ is called a quasi-contraction if there exists $q \in[0,1)$ such that

$$
\begin{aligned}
& p(T x, T y) \leq q \max \{p(x, y), p(x, T x), p(y, T y), p(x, T y), p(y, T x)\} \\
& \quad \text { for all } x, y \in X .
\end{aligned}
$$

One of nice fixed point theorems in rectangular metric spaces is as follows.

Theorem 1 ([4], Theorem 2.2). Let $(X, d)$ be a T-orbitally complete rectangular metric space and $T: X \longrightarrow X$ a quasi-contraction on $X$. Then

(1) $T$ has a unique fixed point $u \in X$.

(2) $\lim _{n \rightarrow \infty} T^{n} x=u$, for every $x \in X$.

(3) $d\left(T^{n} x, u\right) \leq \frac{q^{n}}{1-q} \max \left\{d(x, T x), d\left(x, T^{2} x\right)\right\}$, for all $n \in \mathbb{N}$.

A relation between a partial rectangular metric space and some rectangular metric spaces was stated in [12] is as follows.

Theorem 2. Let $(X, p)$ be a partial rectangular metric space and

$$
p^{r}(x, y)=2 p(x, y)-p(x, x)-p(y, y)
$$

for all $x, y \in X$. Then we have

(1) [12, Proposition 1] $p^{r}$ is a rectangular metric on $X$. 
(2) [12, Lemma 1] $\lim _{n \rightarrow \infty} x_{n}=x$ in $(X, p)$ if and only if $\lim _{n \rightarrow \infty} x_{n}=x$ in $\left(X, p^{r}\right)$.

(3) $\left[12\right.$, Lemma 2] A sequence $\left\{x_{n}\right\}$ is Cauchy in $(X, p)$ if and only if it is Cauchy in $\left(X, p^{r}\right)$.

\section{MAin RESUlts}

First, we give some new notions in partial rectangular metric spaces.

Definition 6. Let $(X, p)$ be a partial rectangular metric space.

(1) A sequence $\left\{x_{n}\right\} \subset X$ is called 0-Cauchy if $\lim _{n, m \rightarrow \infty} p\left(x_{n}, x_{m}\right)=0$.

(2) $(X, p)$ is called 0 -complete if for each 0-Cauchy sequence $\left\{x_{n}\right\}$ in $X$, there exists some $x \in X$ such that

$$
\lim _{n \rightarrow \infty} p\left(x_{n}, x\right)=p(x, x)=\lim _{n, m \rightarrow \infty} p\left(x_{m}, x_{n}\right)=0 .
$$

Lemma 1. Let $(X, p)$ be a partial rectangular metric space.

(1) If $(X, p)$ is complete, then it is 0-complete.

(2) If $x_{n} \neq x, y_{n} \neq y, x_{n} \neq y_{n}$ for all $n \in \mathbb{N}$ and

$$
\lim _{n \rightarrow \infty} x_{n}=x, \lim _{n \rightarrow \infty} y_{n}=y, p(x, x)=p(y, y)=0
$$

then $\lim _{n \rightarrow \infty} p\left(x_{n}, y_{n}\right)=p(x, y)$.

Proof. (1). It is straightforward from the definition.

(2). For each $n \in \mathbb{N}$, we have

$$
p(x, y)-p\left(x_{n}, y_{n}\right)
$$

(1) $\leq p\left(x, x_{n}\right)+p\left(x_{n}, y_{n}\right)+p\left(y_{n}, y\right)-p\left(x_{n}, x_{n}\right)-p\left(y_{n}, y_{n}\right)-p\left(x_{n}, y_{n}\right)$

$$
\begin{aligned}
\leq & p\left(x, x_{n}\right)+p\left(y, y_{n}\right), \\
& p\left(x_{n}, y_{n}\right)-p(x, y)
\end{aligned}
$$

$(2) \leq p\left(x_{n}, x\right)+p(x, y)+p\left(y, y_{n}\right)-p(x, x)-p(y, y)-p(x, y)$

$$
\leq p\left(x_{n}, x\right)+p\left(y, y_{n}\right) \text {. }
$$

From (1) and (2) we have

$$
\left|p(x, y)-p\left(x_{n}, y_{n}\right)\right| \leq p\left(x_{n}, x\right)+p\left(y, y_{n}\right) .
$$

Taking the limits as $n \rightarrow \infty$ in (3), we get $\lim _{n \rightarrow \infty} p\left(x_{n}, y_{n}\right)=p(x, y)$.

The following example shows that the converse of Lemma 1.(1) need not hold.

Example 3. Let $X=(0,1)$ and $p(x, y)=|x-y|+2$ for all $x, y \in X$. Then we have

(1) $p$ is a partial rectangular metric.

(2) $(X, p)$ is 0 -complete.

(3) $(X, p)$ is not complete. 
Proof. (1). It is easy to check that $p$ satisfies conditions (1) to (4) in Definition 3. Now, we will show that $p(x, y) \leq p(x, w)+p(w, z)+p(z, y)-$ $p(w, w)-p(z, z)$ for all distinct points $w, z \in X \backslash\{x, y\}$.

Indeed, for all distinct points $w, z \in X \backslash\{x, y\}$, we have

$$
\begin{aligned}
p(x, y) & =|x-y|+2 \\
& \leq|x-w|+|w-z|+|z-y|+2 \\
& =|x-w|+2+|w-z|+2+|z-y|+2-2-2 \\
& =p(x, w)+p(w, z)+p(z, y)-p(w, w)-p(z, z)
\end{aligned}
$$

Hence, $p$ is a partial rectangular metric on $X$.

(2). Since $p(x, y) \geq 2$ for all $x, y \in X,(X, p)$ has no any 0-Cauchy sequence. This proves that $(X, p)$ is 0 -complete.

(3). We have that

$$
\lim _{n, m \rightarrow \infty}\left(\frac{1}{2 n}, \frac{1}{2 m}\right)=\lim _{n, m \rightarrow \infty}\left(\left|\frac{1}{2 n}-\frac{1}{2 m}\right|+2\right)=2 .
$$

Then $\left\{\frac{1}{2 n}\right\}$ is a Cauchy sequence in $(X, p)$. Suppose to the contrary that $\lim _{n \rightarrow \infty} \frac{1}{2 n}=x$ in $(X, p)$. We have that

$$
\lim _{n \rightarrow \infty} p\left(\frac{1}{2 n}, x\right)=\lim _{n \rightarrow \infty}\left(\left|\frac{1}{2 n}-x\right|+2\right)=p(x, x)=2 .
$$

It implies that $x=0 \in X=(0,1)$. It is a contradiction. Then $\left\{\frac{1}{2 n}\right\}$ is not convergent in $(X, p)$. Therefore, $(X, p)$ is not complete.

The main results of the paper are as follows.

Theorem 3. Let $(X, p)$ be a partial rectangular space and

$$
d_{p}(x, y)= \begin{cases}0, & \text { if } x=y \\ p(x, y), & \text { if } x \neq y\end{cases}
$$

Then we have

(1) $d_{p}$ is a rectangular metric on $X$.

(2) The partial rectangular metric space $(X, p)$ is 0 -complete if and only if the rectangular metric space $\left(X, d_{p}\right)$ is complete.

Proof. (1). It is easy to see that $d_{p}$ is a rectangular metric on $X$.

(2). Suppose that the partial rectangular metric space $(X, p)$ is 0 -complete. For each Cauchy sequence $\left\{x_{n}\right\}$ in the rectangular metric space $\left(X, d_{p}\right)$, we may assume that $x_{n} \neq x_{m}$ for all $n \neq m \in \mathbb{N}$. Then we have

$$
\lim _{n, m \rightarrow \infty} p\left(x_{n}, x_{m}\right)=\lim _{n, m \rightarrow \infty} d_{p}\left(x_{n}, x_{m}\right)=0 .
$$


This proves that $\left\{x_{n}\right\}$ is a 0 -Cauchy sequence in the 0 -complete partial rectangular metric space $(X, p)$. Then there exists $x \in X$ such that

$$
\lim _{n \rightarrow \infty} p\left(x_{n}, x\right)=p(x, x)=\lim _{n, m \rightarrow \infty} p\left(x_{n}, x_{m}\right)=0 .
$$

It implies that

$$
\lim _{n \rightarrow \infty} d_{p}\left(x_{n}, x\right)=\lim _{n \rightarrow \infty} p\left(x_{n}, x\right)=0 .
$$

This proves that $\lim _{n \rightarrow \infty} x_{n}=x$ in the rectangular metric space $\left(X, d_{p}\right)$. Then the rectangular metric space $\left(X, d_{p}\right)$ is complete.

Conversely, suppose that the rectangular metric space $\left(X, d_{p}\right)$ is complete. For each 0-Cauchy sequence $\left\{x_{n}\right\}$ in the partial rectangular metric space $(X, p)$, we may assume that $x_{n} \neq x_{m}$ for all $n \neq m \in \mathbb{N}$. We have $\lim _{n, m \rightarrow \infty} p\left(x_{n}, x_{m}\right)=0$. It implies that

$$
\lim _{n, m \rightarrow \infty} d_{p}\left(x_{n}, x_{m}\right)=\lim _{n, m \rightarrow \infty} p\left(x_{n}, x_{m}\right)=0 .
$$

Thus, $\left\{x_{n}\right\}$ is a Cauchy sequence in the complete rectangular metric space $\left(X, d_{p}\right)$. Then there exists $x \in X$ such that $\lim _{n \rightarrow \infty} d_{p}\left(x_{n}, x\right)=0$. It implies that $\lim _{n \rightarrow \infty} p\left(x_{n}, x\right)=0$. Also, we have

$$
0 \leq p(x, x) \leq \lim _{n \rightarrow \infty} p\left(x, x_{n}\right)=0 .
$$

Therefore,

$$
\lim _{n \rightarrow \infty} p\left(x_{n}, x\right)=p(x, x)=\lim _{n, m \rightarrow \infty} p\left(x_{n}, x_{m}\right)=0 .
$$

Then the partial rectangular metric space $(X, p)$ is 0 -complete.

Theorem 4. Let $(X, p)$ be a partial rectangular metric space and $T: X \longrightarrow$ $X$ be a quasi-contraction map on the partial rectangular metric space $(X, p)$. Then $T$ is a quasi-contraction map on the rectangular metric space $\left(X, d_{p}\right)$.

Proof. Since $T$ is a quasi-contraction map on $(X, p)$, there exists $q \in[0,1)$ such that

$$
p(T x, T y) \leq q \max \{p(x, y), p(x, T x), p(y, T y), p(x, T y), p(y, T x)\}
$$

for all $x, y \in X$. If $T x=T y$, then $d_{p}(T x, T y)=0$. It implies that

$$
d_{p}(T x, T y) \leq q \max \left\{d_{p}(x, y), d_{p}(x, T x), d_{p}(y, T y), d_{p}(x, T y), d_{p}(y, T x)\right\} .
$$

If $T x \neq T y$, then $x \neq y$. It implies that $p(x, y)=d_{p}(x, y)$. Moreover, if $x=T x$, then $p(x, T x) \leq p(x, y)=d_{p}(x, y)$, otherwise, $p(x, T x)=d_{p}(x, T x)$. Also, if $y=T x$, then

$$
p(y, T x) \leq p(y, x)=d_{p}(x, y) .
$$


Otherwise, $p(y, T x)=d_{p}(y, T x)$. Therefore,

$$
\begin{aligned}
d_{p}(T x, T y) & =p(T x, T y) \\
& \leq q \max \{p(x, y), p(x, T x), p(y, T y), p(x, T y), p(y, T x)\} \\
& =q \max \left\{d_{p}(x, y), p(x, T x), p(y, T y), p(x, T y), p(y, T x)\right\} \\
& \leq q \max \left\{d_{p}(x, y), d_{p}(x, T x), d_{p}(y, T y), d_{p}(x, T y), d_{p}(y, T x)\right\} .
\end{aligned}
$$

By the above, $T$ is a quasi-contraction map on the rectangular metric space $\left(X, d_{p}\right)$.

From Theorem 4, we show that the result in [12] is a consequence of Theorem 1.

Corollary 1 ([12], Theorem 6). Let $(X, p)$ complete partial rectangular metric space and $T: X \longrightarrow X$ be a quasi-contraction on $X$. Then $T$ has a unique fixed point $u \in X$ and $p(u, u)=0$.

Proof. Let $d_{p}$ be defined as in Theorem 3. It follows from Theorem 3 and Theorem 4 that $\left(X, d_{p}\right)$ is a complete rectangular metric space and $T: X \longrightarrow$ $X$ is a quasi-contraction on $\left(X, d_{p}\right)$. By Theorem $1, T$ has a unique fixed point $u \in X$. The fact $p(u, u)=0$ is easy to get from the assumption.

Remark 1. By using argument in the proof of Corollary 1, we may transform many fixed point theorems on rectangular metric spaces in the literature, such as $[4,5,6]$ and references therein, to partial rectangular metric spaces.

\section{REFERENCES}

[1] A. Al-Bsoul, A. Fora, and A. Bellour, Some properties of generalized metric spaces and fixed point theory, Mate. Stu. (2010), 85 - 91.

[2] A. Branciari, A fixed point theorem of Banach-Caccioppoli type on a class of generalized metric spaces, Publ. Math. Debrecen 57 (2000), no. 1-2, 31 - 37.

[3] R. H. Haghi, S. Rezapour, and N. Shahzad, Be careful on partial metric fixed point results, Topology Appl. 160 (2013), no. 3, 450 - 454.

[4] L. Kikina and K. Kikina, On fixed point of a Ljubomir Ćirić quasi-contraction mapping in generalized metric spaces, Publ. Math. Debrecen (2013), in press.

[5] L. Kikina and K. Kikina, A fixed point theorem in generalized metric spaces, Demonstratio Math. XLVI (2013), no. 1, 181 - 190.

[6] W. A. Kirk and N. Shahzad, Generalized metrics and Caristi's theorem, Fixed Point Theory Appl. 2013:129 (2013), 1 - 9.

[7] B. K. Lahiri and P. Das, Fixed point of a Ljubomir Ciric's quasi-contraction mapping in a generalized metric space, Publ. Math. Debrecen 61 (2002), no. 3-4, 589 - 594.

[8] S. K. Malhotra, S. Radenovic, and S. Shukla, Some fixed point results without monotone property in partially ordered metric-like spaces, J. Egyptian Math. Soc. (2013), $1-7$, in press. 
[9] S. G. Matthews, Partial metric topology, Ann. New York Acad. Sci. 728 (1994), 183 $-197$.

[10] I. A. Rus, Fixed point theory in partial metric spaces, An. Univ. Vest Timiş. Ser. Mat.-Inform. XLVI (2008), no. 2, 149 - 160.

[11] B. Samet, C. Vetro, and F. Vetro, From metric spaces to partial metric spaces, Fixed Point Theory Appl. 2013:5 (2013), 1 - 11.

[12] S. Shukla, Partial rectangular metric spaces and fixed point theorems, Sci. World J. (2013), $1-12$, in press.

[13] S. Shukla, S. Radenović, and Z. Kadelburg, Some fixed point theorems for ordered $f$ generalized contractions in 0-f-orbitally complete partial metric spaces, Theory Appl. Math. Comput. Sci. 4 (2014), no. 1, $87-98$.

[14] S. Shukla, S. Radenović, and V. C. Rajić, Some common fixed point theorems in 0- $\sigma$-complete metric-like spaces, Vietnam J. Math. 41 (2013), no. 3, 341 - 352.

NGUYen VAN Dung

FACUlty of Mathematics and Information

Technology Teacher Education

Dong Thap UnIVERSiTy

Cao Lanh City, Dong Thap Province 871200

VIET NAM

E-mail address: nvdung@dthu.edu.vn

nguyendungtc@yahoo.com

\section{Vo Thi Le Hang}

Journal of SCIENCE

Dong Thap UnIVERSiTy

Cao Lanh City, Dong Thap Province 871200

VIET NAM

E-mail address: vtlhang@dthu.edu.vn 